\title{
ARTICLE Returning to cataract surgery after a hiatus: a UK survey report
}

\author{
Laura Maubon (iD ${ }^{1,2}$, Paul Nderitu ${ }^{3}$ and David P. S. O'Brart ${ }^{1,4}$ \\ (c) The Author(s), under exclusive licence to The Royal College of Ophthalmologists 2021
}

\begin{abstract}
OBJECTIVES: Cataract surgeons may periodically take time away from operating which can lead to skills fade. There is a paucity of research investigating the experiences of returning cataract surgeons and how different individual circumstances impact on their return. Our aim was to investigate the subjective experiences of UK ophthalmologists simultaneously returning to surgery following the nationwide elective surgical hiatus due to the Covid-19 pandemic.
\end{abstract}

METHODS: An online survey was nationally distributed between 01/09/2020 and 29/10/2020 to registered UK ophthalmologists. Participants indicating a surgical hiatus of 8 weeks or more were included.

RESULTS: 232 of 264 responses were analysed. Covid-19 was the most frequent reason for a surgical hiatus (median 15 weeks). Perceived operating difficulties were found in $29.1 \%$. Transient anxiety $(51.7 \%)$, reduced confidence, and perceived increased surgical time were commonplace. Trainees and females were more likely to encounter negative experiences $(p<0.001)$ and barriers to resource accessibility. Eyesi ${ }^{\circ}$ and online videos were the most available and accessed pre-return resources. Childcare was five times more likely to present as a barrier to resource access for females than males.

CONCLUSIONS: Technical skills fade such as capsulorhexis difficulties were commonly perceived by trainee surgeons in addition to transient anxiety, reported in more than half of all surgeons following a hiatus as short as 8 weeks.

Eyesi simulation offers the potential to negate technical de-skilling. Few had formal return plans or awareness of RCOphth guidance. We believe there is scope for more specialised and targeted support for future returning cataract surgeons thereby optimising patient care.

Eye (2022) 36:1761-1766; https://doi.org/10.1038/s41433-021-01717-5

\section{INTRODUCTION}

Cataract surgery is the most frequently performed elective surgical procedure globally, with an estimated 452,000 procedures undertaken in the UK during 2018-19 [1]. With modern, microscopic, micro-incisional surgical techniques, visual and refractive outcomes are excellent with almost $95 \%$ of eyes achieving 0.3 LogMAR corrected visual acuity or better in eyes without co-pathology [2]. Therefore, whilst it demands a high level of micro-surgical skills, cataract surgery is not only the most common but also one of the most successful in modern medicine, with increasingly higher patient and surgeon outcome expectations [3].

Surgeons may need to periodically take breaks from operating for a variety of reasons. In March 2020, the unprecedented Covid-19 pandemic resulted in a nationwide elective surgery hiatus with many ophthalmologists forced into a surgical break [3-7]. Surgical breaks have been shown to lead to technical skills fade [8]. Observational studies amongst medical practitioners suggest that beyond a six-month interval, de-skilling, particularly fine-motor skills, occurs rapidly at the outset followed by a slower skill degradation over time [9-11]. The rate of skill loss varies between individuals and is dependent on many mitigating factors $[8,12]$.

In addition to technical skill loss associated with such surgical breaks, other non-technical factors such as increased stress, anxiety, and lost confidence can occur, which may additionally impact on a surgeons' performance [13-15]. Since reduced performance may compromise patient safety, it is essential to understand better the impact of any surgical hiatus. This is particularly important in relation to cataract surgery, given the frequency at which it is undertaken and the high technical microsurgical skills that modern cataract surgery demands [1-3].

There is a paucity of research and published literature to understand how factors such as the length of time away from undertaking surgery, return resource availability and utilisation, personal circumstances on return, and prior surgical exposure can impact ophthalmologists' returning to cataract surgery experiences and outcomes [15]. Whilst general guidelines exist, they lack any specificity and compulsion, and as such it is unknown whether ophthalmologists are aware or even utilise them [16-20]. This is in great contrast to comparable 'high reliability' professions such as aviation, which may also experience similar trends of technical skills degradation following breaks and have carefully considered the possible resultant implications for passenger safety [21]. Unlike ophthalmologists, commercial pilots are subject to compulsory checklists, in addition to mental and physical screening before recommencing their technical duties. A minimum of three take off and landings within 90 days are specified as an absolute requirement, which was altered to 1 in 60 days because of Covid-19 related skills fade [22]. In addition, required prescribed hours of simulation, combined with supervised flights, are well established for commercial pilots returning to flying after extended breaks $[22,23]$. In terms of patient safety and knowing that surgical breaks lead to skill fade, it would seem prudent that

\footnotetext{
${ }^{1}$ Guy's and St Thomas' NHS Foundation Trust, London, UK. ${ }^{2}$ Moorfields Eye Hospital NHS Foundation Trust, London, UK. ${ }^{3}$ King's College Hospital, NHS Foundation Trust, London,
} UK. ${ }^{4}$ King's College London, London, UK. ${ }^{凶}$ email: lauramaubon@nhs.net 
ophthalmology attempts to learn lessons from the aviation industry by investigating factors that may improve returns from surgical breaks, with perhaps a view to the development of more robust guidelines so that the outcomes of cataract surgery are optimised, and patient safety maintained.

To address these issues and given that the Covid-19 pandemic with periods of cessation of elective surgery during the first lockdown period in the UK from March to June 2020 provided an opportunity to study the subjective effects of time away from cataract surgery in ophthalmic surgeons on mass, we constructed and undertook this study. Our aim was to investigate the experiences and problems encountered by ophthalmic surgeons returning to cataract surgery following a minimum hiatus of 8 weeks, through a nationally distributed online survey, with the aim of identifying factors that are important in facilitating a safe return to elective cataract surgery.

\section{METHODS}

\section{Ethics}

All participants were required to consent to the publication and analysis of anonymized aggregate online survey data. The Health Research Authority (HRA) decision tool confirmed ethical approval was not required for this study [24]. The survey was registered locally at Guys and St Thomas NHS trust.

\section{Survey design and participants}

The survey questions were informed by a literature search of peerreviewed databases and published articles for terms relating to return to practice, surgery, and skills fade [8-23]. Draft questions were iteratively discussed and amended by authors LM and PN with senior oversight from DO'B. Bias was minimised through careful question selection, ordering, and the use of capitalisation for emphasis. A variety of single, multi-selection, multi-checkbox, and response validated date and time question formats were selected based on their suitability and ease of use. Final questions were used to develop an online survey using Google Forms ${ }^{\mathrm{Tm}}$. The draft survey was reviewed and approved by The Royal College of Ophthalmologists prior to finalisation. The survey was piloted and tested to ensure all responses were correctly recorded. Survey questions are available in the supplementary data.

The survey was distributed via email by The Royal College of Ophthalmologists to all non-retired UK members on 1st September 2020 (approximately 3 months after the first Covid-19 lockdown restrictions eased in the UK) to all UK ophthalmologists with a valid registration to maximise responses. Responses were collected until the 29th October 2020. Participants responses were included where they indicated a surgical hiatus of 8 weeks or more, followed by a subsequent return to cataract surgery.

\section{Statistical analysis}

Continuous variables are presented as means (+/ - standard deviation, SD). Categorical variables are reported as counts and percentages. For multi-response questions (resource utilisation, access barriers, operating difficulties, and subjective experiences) reported percentages are denominated by the total number of responses. The chi-squared test $\left(x^{2}\right)$ was used to ascertain the level of significance. Multi-response questions were dichotomised into no/yes groups for the presence of $\geq 1$ affirmative responses to ensure the mutually exclusive assumption of the $x^{2}$ test was not violated. All tests were performed using SPSS v.25, Chicago, IL.

\section{RESULTS}

From 264 entries, 232 unique entries fulfilled the inclusion criteria and were eligible for analysis. Given the survey sample size $(n=$ 232), a 95\% confidence interval and an estimated 1900-2200 UKbased ophthalmic surgeons (estimate calculated from RCOphth census) gave a $6 \%$ margin of error for study responses (Survey Monkey Calculator Inc, USA) [25, 26]. The mean age of respondents was 46 years (SD: 11 years). Trainee/fellow gender ratios were 52:48 for males and females compared to 72:28 for consultants and SAS. One in three respondents had performed
Table 1. Demographics and cataract surgery experience of survey participants.

\begin{tabular}{|c|c|c|}
\hline Demographics & Count or [Mean] & $\%$ or (SD) \\
\hline \multicolumn{3}{|l|}{ Age } \\
\hline Years & [46] & (11) \\
\hline \multicolumn{3}{|l|}{ Gender } \\
\hline Male & 154 & 66.4 \\
\hline Female & 78 & 33.6 \\
\hline \multicolumn{3}{|l|}{ Ethnicity } \\
\hline White & 102 & 44.0 \\
\hline Black/Black British & 7 & 3.0 \\
\hline Asian/Asian British & 84 & 36.2 \\
\hline Mixed/Multi-Ethnicity & 6 & 2.6 \\
\hline Other Ethnic Group & 23 & 9.9 \\
\hline Not-disclosed & 10 & 4.3 \\
\hline \multicolumn{3}{|l|}{ Seniority } \\
\hline Consultant or SAS & 172 & 74.1 \\
\hline Trainee or Fellow & 60 & 25.9 \\
\hline \multicolumn{3}{|l|}{ Practice } \\
\hline NHS & 132 & 56.9 \\
\hline NHS and Private & 83 & 35.8 \\
\hline Private & 11 & 4.7 \\
\hline N/A or Undeclared & 6 & 2.6 \\
\hline \multicolumn{3}{|l|}{ Cataracts performed } \\
\hline$<100$ & 12 & 5.2 \\
\hline $100-249$ & 13 & 5.6 \\
\hline $250-349$ & 9 & 3.9 \\
\hline $350-499$ & 15 & 6.5 \\
\hline $500-999$ & 34 & 14.7 \\
\hline $1000-5000$ & 71 & 30.6 \\
\hline$>5000$ & 78 & 33.6 \\
\hline
\end{tabular}

SD standard deviation, NHS National Health Service.

over 5000 cataracts whilst $14.7 \%$ had performed less than 350 cataract operations (Table 1).

Most respondents (95.6\%) cited Covid-19 pandemic restrictions as their primary reason for their most recent surgical hiatus which lasted a median of 15 weeks (interquartile range: $12-18$ weeks). Other reasons for breaks in cataract surgery were research $(1.8 \%$, median 38 weeks), maternity leave (1.3\%, median 36 weeks), and other (1.3\%, median 18 weeks). Approximately $25 \%(n=57)$ of respondents had one or more previous breaks away from cataract surgery prior to the Covid-19 pandemic; however, of these, only $3.1 \%$ reported two or more time-outs.

\section{Resources available, accessed, and utilised}

The Eyesi (VR Magic, Germany) simulator and online videos were available to approximately $50 \%$ of respondents with $25-30 \%$ reporting eLearning and wet/dry labs availability (Fig. 1). Response for accessed resources was generally lower than those for availability, with Eyesi $(29.3 \%)$ and online videos (45\%) being most frequently accessed. Low complexity lists (37.1\%), Eyesi simulator (37.9\%), and wet/dry labs (24.6\%) were the most highly requested resources. Some respondents reported having no local resources available $(22.5 \%)$ or did not request additional resources $(32.3 \%)$. Low complexity lists had the lowest reported barriers to access (53\%) (Fig. 1). Female surgeons and trainees were significantly more likely to report resource availability and to have accessed resources, however, they were more likely to request additional resources and 


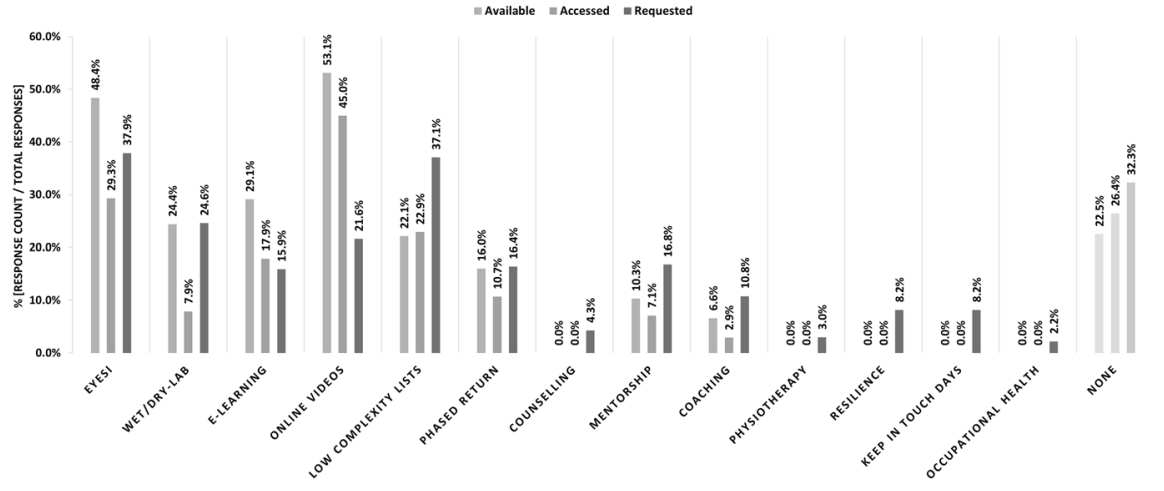

Fig. 1 Resources available, accessed, and requested. Eyesi: virtual cataract surgery simulator.

reported significantly higher barriers to accessing resources (Table 2). Female surgeons were significantly more likely to cite childcare/ home-schooling barriers (20\%) compared to male surgeons $(4 \%)$, with time and inadequate facilities also more commonly reported barriers to resource access for female compared to male surgeons (Supplementary Data: Table 1).

\section{Returning to cataract surgery after a hiatus}

Only $11.6 \%$ of respondents reported that a formal plan was made prior their return cataract surgery, whilst $31.5 \%$ reported an awareness of return to practice guidelines, of which $63 \%$ found them useful. Operating difficulties were frequently encountered after returning to cataract surgery (29.1\%), with main incision and capsulorrhexis construction, phacoemulsification, and posterior capsular rupture (PCR) management the most frequently reported difficulties (Fig. 2). Operating difficulties were significantly associated with trainee status $(p<0.001)$ but not gender. Commonly reported negative experiences after returning to cataract surgery were transient anxiety, a perceived increase in operating time, and reduced confidence in operating ability. Negative experiences were significantly more common amongst female and trainee surgeons $(p<0.001)$, especially a perceived increase in operating time and reduced confidence in operating ability (Fig. 3). There was a significant association between negative experiences and cataract surgery experience $(p<0.001)$. The proportion of ophthalmic surgeons with up to 7 years' cataract surgery experience reporting one or more negative experiences was $96 \%$, compared to $61.9 \%$ amongst surgeons with an excess of 20 years' experience. Protective factors against negative experiences included older age, male gender, and prior surgical experience.

\section{DISCUSSION}

The survey response rate ( $12 \%$ of UK ophthalmic surgeons) compares favourably to other nationally distributed surveys $[15,27,28]$. Demographic data for age, gender, trainee/fellow/ consultant/SAS status matched well with RCOphth census reports (Table 1) [25]. Trainees accounted for $26 \%$ of the cohort, which correlates with the $25 \%$ estimate from the national census. Most survey respondents (85.4\%) had performed the minimum number of surgeries (350 cataract operations) required prior to the attainment of the certificate of completion of training (CCT) in the UK [29]. Two-thirds of respondents were unaware of any RCOphth guidelines and only 1 in 9 people had a formal plan for returning to surgery, which suggests that there is a need to improve awareness and develop tailored guidelines.

\section{Subjective experiences}

Negative experiences were reported amongst all groups with reduced confidence, time inefficiency, and transient anxiety common-place, and with trainees much more commonly affected (Table 2). Few ophthalmologists reported having no negative experiences, especially trainees, with a multitude of experiences reported including diminished abilities, increased work stress, anxiety, intraoperative complications, and frustration whilst operating. This suggests a surgical hiatus as short as 8 weeks may trigger these negative perceptions. Over-learning, the concept pertaining to having extensive knowledge, more than the required minimum, has been shown to be protective against de-skilling [30]. This concept may explain the survey observations, that more experienced surgeons are better protected from negative experiences [30]. Junior surgeons may underreport negative experiences compared to more senior trainees because of unconscious incompetence, a recognised learning stage [31].

\section{Accessibility and availability of simulation resources}

Trainees were significantly more likely to report the availability of and access resources, but they also reported more access barriers compared to consultants/SAS surgeons (Table 2). Trainees in registrar years tended to report better awareness and utilisation of resources, which may represent a more independent and proactive stage of technical skill acquisition. The most desired resources were Eyesi ${ }^{\oplus}$ and access to low complexity lists. Although Eyesi ${ }^{\circ}$ was reportedly available to $48 \%$ of respondents, only $29 \%$ accessed the surgical simulator, citing Covid-19, distance to the site of the simulator, and time as key barriers. The proactive organisation between the RCOphth, ophthalmic surgeons, administrators, and managers could overcome some of these barriers. It is possible that low complexity lists might have been less achievable during the pandemic recovery because of more visually significant and complex cataracts requiring more timely surgical intervention once services resumed.

Females account for $50 \%$ of the trainee ophthalmologist population and just under a third of the post CCT populous. Interestingly, female surgeons were more likely to have knowledge of available learning resources, access these resources, request resources, identify barriers to accessing resources and report negative subjective experiences on their return (Table 2 ). In part, this might be explained by a higher frequency of females who had taken prior time out, and who may therefore be aware of beneficial resources. The RCOphth census confirms that maternity leave is the most common reason for time out, accounting for $59.6 \%$ of all deanery trainee absences, followed by $21.3 \%$ for out of programme activity, $12.8 \%$ for research, and $6.4 \%$ long term sickness [25]. Childcare responsibilities were cited as barrier to learning resources by a causative factor of five times more when compared with their male colleagues (Supplementary Data: Table 1). Since female trainees returning from maternity leave are likely to have encountered prolonged breaks, confounded by new childcare responsibilities and additional unknown personal circumstances, this montage of mitigating factors could make 
Table 2. Demographic factors associated with resource utilisation, operating difficulties, and negative experiences after returning to cataract surgery.

\begin{tabular}{|c|c|c|c|c|c|c|}
\hline \multirow[t]{2}{*}{ Factor } & \multicolumn{2}{|l|}{ Gender } & \multirow[t]{2}{*}{$p$-value } & \multicolumn{2}{|l|}{ Seniority } & \multirow[t]{2}{*}{$p$-value } \\
\hline & $M \% n=154$ & $F \% n=78$ & & $\begin{array}{l}\text { Consultant or } \\
\text { SAS } \% n=172\end{array}$ & $\begin{array}{l}\text { Trainee or } \\
\text { Fellow } \% n=60\end{array}$ & \\
\hline \multicolumn{7}{|c|}{$\geq 1$ Available resources } \\
\hline No & 32.5 & 19.2 & \multirow[t]{2}{*}{0.034} & 32.0 & 15.0 & \multirow[t]{2}{*}{0.009} \\
\hline Yes & 67.5 & 80.8 & & 67.4 & 85.0 & \\
\hline \multicolumn{7}{|c|}{$\geq 1$ Accessed resources } \\
\hline Yes & 38.3 & 57.7 & 0.005 & 40.1 & 58.3 & 0.015 \\
\hline \multicolumn{7}{|c|}{$\geq 1$ Requested resources } \\
\hline No & 39.0 & 17.9 & \multirow[t]{2}{*}{0.001} & 37.2 & 16.7 & \multirow[t]{2}{*}{0.003} \\
\hline Yes & 61.0 & 82.1 & & 62.8 & 83.3 & \\
\hline \multicolumn{7}{|c|}{$\geq 1$ Resource access barriers } \\
\hline Yes & 20.8 & 30.8 & 0.093 & 16.3 & 46.7 & $<0.001$ \\
\hline \multicolumn{7}{|c|}{$\geq 1$ Negative experiences } \\
\hline No & 31.8 & 9.0 & \multirow[t]{2}{*}{$<0.001$} & 30.2 & 6.7 & \multirow[t]{2}{*}{$<0.001$} \\
\hline Yes & 68.2 & 91.0 & & 68.8 & 93.3 & \\
\hline
\end{tabular}

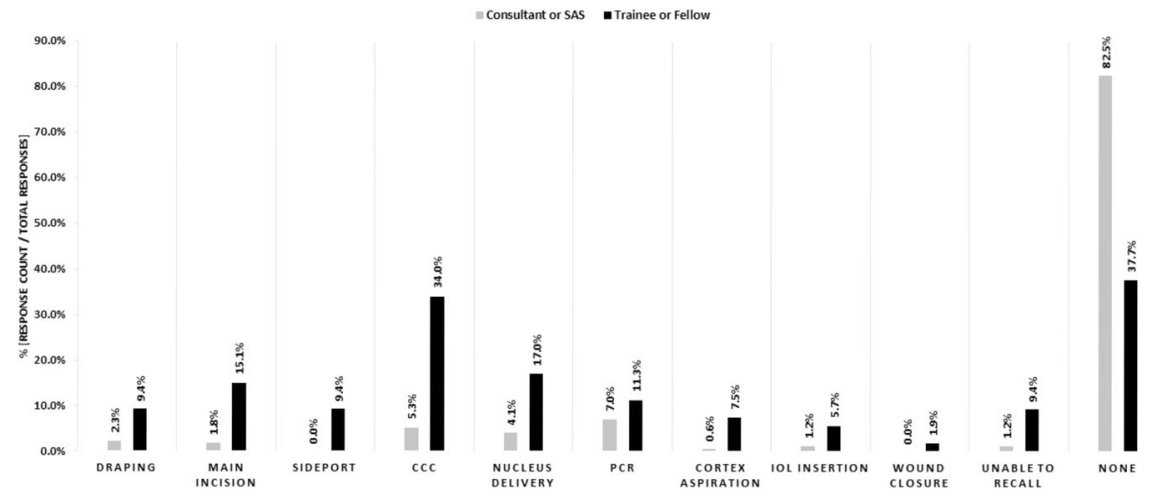

Fig. 2 Operating difficulties after returning to cataract surgery, consultants vs trainees. The response rate for each surgical stay is demonstrated in light grey for consultant and SAS surgeons and dark grey bars for trainees and fellows.

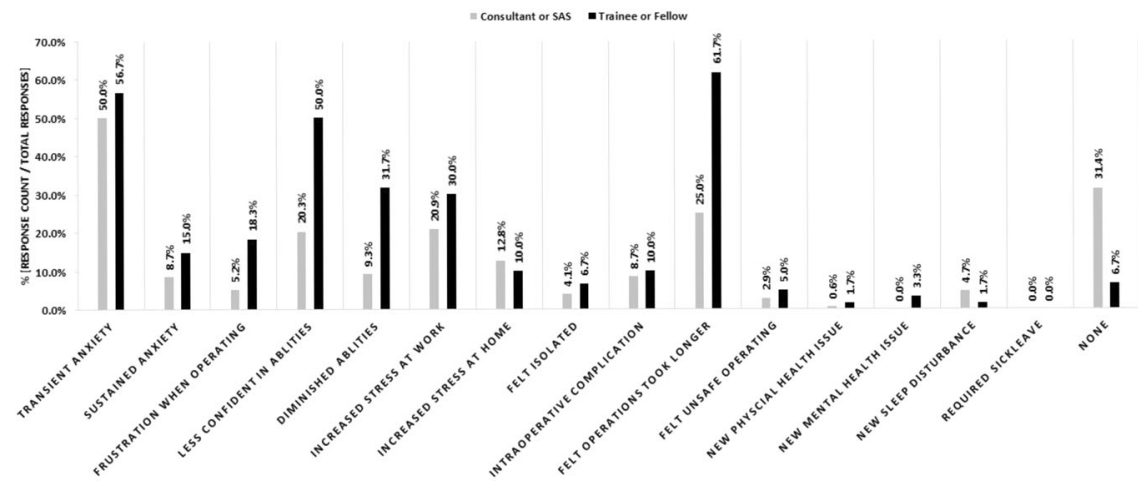

Fig. 3 Negative experiences after returning to cataract surgery, consultants vs trainees. The response frequency for each subjective negative experience encountered is grouped for consultants and SAS in light grey and trainees and fellows in dark grey. 
surgical returns more challenging. As such, targeted guidance for this sub-group might be beneficial and could include accessible simulation resources, more flexible rotas, mentorship, and formal work plans.

\section{Operative difficulties and simulation}

Subjective operative difficulties were experienced by $39.2 \%$ of respondents. There was no difference reported between gender, but it was associated with trainee status and reduced surgical experience. The most reported difficulties were with the main incision, capsulorhexis, phacoemulsification, and PCR. This supports published literature that describes capsulorhexis and phacoemulsification as the two most difficult stages of cataract surgery to learn and further highlights a lack of confidence amongst trainees managing PCR [32-34]. Since poor management of PCR may result in significant visual morbidity, refresher training in PCR management should be an important focus for returning surgeons [35]. The Eyesi ${ }^{\circledR}$ simulator can provide the simulation of capsulorhexis, phacoemulsification, and PCR management. It has also been shown to reduce PCR rates amongst 1 st and 2nd-year surgeons [36, 37]. Since skill repetition can result in improved surgical confidence and improved time efficiency, access to an Eyesi ${ }^{\circledR}$ simulation is a key resource to negate the effects of a surgical hiatus [36]. Overcoming barriers to accessing learning resources could include, better local distribution of equipment, remotely supervised simulation, home-based delivery of simulation, and pre-recorded online training videos. Many of these are currently under development as part of the Covid-19 recovery; updates can be accessed through the RCOphth and UKISCRS websites $[16,38]$.

\section{Limitations}

Covid-19 accounted for more than $95 \%$ of the current surgical time-outs, which should be considered throughout the interpretation of the results. Pandemic-specific factors, such as travel restrictions, may not be generalisable to other reasons for time out. Widespread uncertainty, because of the pandemic, might also influence reported experiences (e.g., anxiety). It is also not possible to infer if the reported PCR complications were higher due to the surgical hiatus, although this is important to investigate and likely an area of future research by the National Ophthalmic Database Audit. It is of note that whilst more trainees reported difficulties with PCR than senior surgeons, these differences were not significant. A significant proportion of respondents $(60 \%)$ had over 13 years' surgical experience and 1 in 3 had performed more than 5000 cataracts which may represent a more senior surgical profile. Equally, senior surgeons were the first to be enrolled back to cataract surgery services following the ease of pandemic restrictions which may influence survey responses.

\section{CONCLUSIONS}

Continuously mounting pressure on public healthcare systems requires a robust workforce to meet demands. It is important to optimise our understanding of the changing workforce to provide the best support and retain highly skilled colleagues while maintaining a focus on patient safety. Our observations have identified common themes amongst cataract surgeons returning from a hiatus, which we believe are applicable to different types of surgical return beyond the Covid-19 pandemic or indeed cataract surgery.

Transient anxiety is widespread and represents a common experience occurring 8 weeks or more after a surgical hiatus. Therefore, the discussion of negative experiences should be encouraged within the framework of supportive supervision and mentorship. Subjective technical skills fade varies between individuals but was highest amongst trainees and those with less surgical experience prior to their hiatus. Self-reported difficulties with capsulorhexis, phacoemulsification, and PCR management are all amenable to good quality simulation [36]. The provision of more flexible, remotely accessible, supervised simulation and increased resource distribution will help to overcome barriers such as childcare constraints and geographical limitations [39]. Currently, there is no compulsory framework for surgical returns for ophthalmology in the UK. Since trainees are more likely to experience challenges, we recommend that a clear checklist, combined with a supervisory meeting, be introduced for ophthalmology trainees returning from intervals of 8 weeks or more. Confident, competent, and supported surgeons will likely provide safer, more efficient, high-quality patient services.

\section{Summary}

What was known before

- Technical skills fade has been observed amongst surgeons following an extended period away from clinical practice. The effects and experiences of returning cataract surgeons following a hiatus, as occurred with the Covid-19 pandemic, are unknown.

What the study adds

- Transient anxiety is a common shared experience across all cataract surgeons returning to surgery following a hiatus of 8 weeks.

- Technical issues with capsulorhexis, phacoemulsification, and posterior capsular rupture are commonly reported on return to cataract surgery, with operating difficulties associated with trainee status.

- One or more negative experiences are commonly reported after returning to cataract surgery following a hiatus with more frequent reporting amongst female and trainee surgeons.

- Trainee status is significantly associated with higher resource utilisation.

- Female surgeons are five times more likely to report barriers to accessing learning resources because of childcare compared with male colleagues.

\section{REFERENCES}

1. Royal College of Ophthalmologists. National Ophthalmology Database Audit, key findings summary. 2019. https://www.nodaudit.org.uk/u/docs/20/ctshjnsvvi/NOD \%20Audit\%20Key\%20Findings\%20Summary\%202020.pdf.

2. Day AC, Donachie PHJ, Sparrow JM, Johnston RL. The royal college of 285 ophthalmologists' national ophthalmology database study of cataract surgery: report 1, visual outcomes and complications. Eye. 2015;4:552-60.

3. Pager CK. Expectations and outcomes in cataract surgery: a prospective test of 2 models of satisfaction. Arch Ophthalmol. 2004;122:1788-92.

4. Spinelli A, Pellino G. COVID-19 pandemic: perspectives on an unfolding crisis. Br J Surg. 2020;107:785-7

5. World Health Organisation. Clinical management of severe acute respiratory infection when novel coronavirus (nCoV) infection is suspected: interim guidance, 25 January 2020. World Health Organization. 2020. https://apps.who.int/iris/handle/ $10665 / 330893$.

6. NHS England and NHS Improvement. Letter to chief executives of all NHS trusts, CCG accountable officers, GP practices and primary care networks, and providers of community health services NHS England and foundation trusts. 2020. https:// www.england.nhs.uk/coronavirus/wpcontent/uploads/sites/52/2020/03/urgentnext-steps-on-nhs-response-to-covid-19-letter-simon-stevens.pdf.

7. British Medical Journal. Covid-19: all non-urgent elective surgery is suspended for at least three months in England. BMJ. 2020;368:m1106.

8. General Medical Council. Skills fade: a review of the evidence that clinical and professional skills fade during time out of practice, and of how skills fade may be measured or remediated. London: GMC; 2014. https://www.gmc-uk. org/-/media/gmc-site-images/about/skills-fade-literature-review-full-report.pdf? $\mathrm{la}=$ en\&hash $=8 \mathrm{E} 0 \mathrm{E} 20 \mathrm{E} 07337 \mathrm{E} 2344$ A5467F9B302C2D83CF2EBA5. 
9. Ahya SN, Barsuk JH, Cohen ER, Tuazon J, McGaghie WC, Wayne DB. Clinical performance and skill retention after simulation-based education for nephrology fellows. Semin Dialysis. 2012;25:470-73.

10. Gold, M, Jensen, $P$, Jedrzkiewicz, M. Motherhood during residency training challenges and strategies. Can Fam Physician. 2005:51:990-1.

11. Hiemstra E, Kolkman W, van de Put MA, Jansen FW. Retention of basic laparoscopic skills after a structured training program. Gynecol Surg. 2009;6:229-35.

12. Maagaard M, Sorensen JL, Oestergaard J, Dalsgaard T, Grantcharov TP, Ottesen BS, et al. Retention of laparoscopic procedural skills acquired on a virtual-reality surgical trainer. Surg Endosc. 2011;25:722-7.

13. Nash EJ, Curry J, Blackburn S. Returning to theatre after an interval. Bull R Coll Surg Engl. 2018;100:277-81.

14. Brightwell A, Minson S, Ward A, Fertleman C. Returning to clinical training after maternity leave. BMJ. 2013;347:f5965.

15. Ho DK, Chiu AKC, Tung SW. Restarting cataract surgery after an extended period out of training: a perspective from the United Kingdom. Eye. 2020. https://doi. org/10.1038/s41433-020-01224-z.

16. Royal College of Ophthalmologists. Mitigating the impact of COVID-19 on ophthalmology training. 2020. https://www.rcophth.ac.uk/wp-content/ uploads/2020/11/Mitigating-the-effects-of-COVID-19-on-ophthalmologists-intraining-November-2020.pdf.

17. Royal College of Anaesthetists. Returning to work after a period of absence. London: RcoA; 2015. https://www.rcoa.ac.uk/system/files/ReturnToWork2015.pdf.

18. Health Education England, NHS. Supported return to training. 2017. https://www. hee.nhs.uk/sites/default/files/documents/Supported\%20Return $\% 20$ to\% 20Training.pdf.

19. Academy of Medical Royal Colleges. Return to practice guidance: 2017 revision. London: AoMRC; 2017. https://www.aomrc.org.uk/wp-content/uploads/2017/06/ Return_to_Practice_guidance_2017_Revison_0617-2.pdf.

20. British medical Association. Returning to clinical practice after absence. 2020. https://www.bma.org.uk/advice-and-support/career-progression/applying-for-ajob/returning-to-clinical-practice-after-absence.

21. Cahillane, MA \& Morin, C. Skills retention in a complex battlefield management system: a pilot study. Yarralumla, Australia: Argos Press Pty Ltd; 2012.

22. European Union Aviation Safety Agency. Guidelines for handling exemptions to flight crew recent experience requirements in the field of commercial air transport operations. 2020. https://www.easa.europa.eu/sites/default/files/dfu/ easa_guidelines-exemption_fc_recency_oro.fc_.100-fcl.060_issue_3_09.07.20. pdf.

23. International Air Transport Association. Guidance for managing pilot training and licensing during COVID-19 operations. 2020. https://www.iata.org/contentassets/ c0f61fc821dc4f62bb6441d7abedb076/iata-guidance-for-managing-pilottraining-licensing-during-covid19.pdf.

24. National Health Service. Health Research Authority decision tool, UK. 2020. http://www.hra-decisiontools.org.uk/ethics/.

25. The Royal College of Ophthalmologists. Workforce Census 2018. 2019. https://www.rcophth.ac.uk/wp-content/uploads/2019/02/RCOphth-WorkforceCensus-2018.pdf.

26. SurveyMonkey Inc. San Mateo, California, USA. 2021. https://www.surveymonkey. co.uk/mp/margin-of-error-calculator/.

27. Dean WH, Grant S, McHugh J, Bowes O, Spencer F. Ophthalmology specialist trainee survey in the United Kingdom. Eye. 2019;33:917-24.

28. Abdelfattah NS, Radwan AE, Sadda SR. Perspective of ophthalmology residents in the United States about residency programs and competency in relation to the International Council of Ophthalmology guidelines. J Curr Ophthalmol. 2016;28: 146-51.

29. The Royal College of Ophthalmologists. Award of the Certificate of Completion of Training (CCT), Surgical Skills SS4. 2021. https://www.rcophth.ac. uk/learningoutcomes/ss4/.

30. Ericsson KA. Chapter 38 The influence of experience and deliberate practice on the development of superior expert performance. In: The Cambridge handbook of expertise and expert performance. Florida, USA: Florida State University; 2006. p. 685-705.

31. Broadwell, M. Teaching for learning (XV1.) Vol 20, no 1-3a. 1969. https://edbatista. typepad.com/files/teaching-for-learning-martin-broadwell-1969-consciouscompetence-model.pdf.

32. Dooley IJ, O'Brien PD. Subjective difficulty of each stage of phacoemulsification cataract surgery performed by basic surgical trainees. J Cataract Refract Surg. 2006;32:604-8.

33. Grinton M, Sandhu J, Shwe-Tin A, Steel DHW, Ting DSJ, North East Trainee Research in Ophthalmology Network (NETRiON). Incidence, characteristics, outcomes and confidence in managing posterior capsular rupture during cataract surgery in the UK: an ophthalmology trainees' perspective. Eye. 2020. https://doi. org/10.1038/s41433-020-1057-z.

34. Turnbull AM, Lash SC. Confidence of ophthalmology specialist trainees in the management of posterior capsule rupture and vitreous loss. Eye. 2016; 30:943-94.

35. Ti S-E, Yang Y-N, Lang SS, Chee SP. A 5-year audit of cataract surgery outcomes after posterior capsule rupture and risk factors affecting visual acuity. Am J Ophthalmol. 2014;157:180-5.

36. Lee R, Raison N, Lau WY, Aydin A, Dasgupta P, Ahmed K. A systematic review of simulation-based training tools for technical and non-technical skills in ophthalmology. Eye. 2020;34:1737-59. https://doi.org/10.1038/s41433-020-0832-1.

37. Ferris JD, Donachie PH, Johnston RL, Barnes B, Martina O, Sparrow M. Royal College of Ophthalmologists' National Ophthalmology Database study of cataract surgery: report 6 . The impact of EyeSi virtual reality training on complications rates of cataract surgery performed by first and second year trainees. $\mathrm{Br} \mathrm{J}$ Ophthalmol. 2019;104:324-9.

38. Royal College of Ophthalmologists. Remotely supervised simulated surgery. 2021. https://www.rcophth.ac.uk/event/remotely-supervised-simulated-surgerywebinar/.

39. Maubon L, Nderitu P, Swampillai AJ. National access to Eyesi ${ }^{\circledR}$ and anterior vitrectomy simulation. Eye. 2020. https://doi.org/10.1038/s41433-020-1107-6.

\section{AUTHOR CONTRIBUTIONS}

$\mathrm{LM}, \mathrm{PN}$ and $\mathrm{DO}$ 'B developed the project concept. $\mathrm{LM}$ and $\mathrm{PN}$ designed the survey questions and online forms. $L M$ and PN helped distribute the survey via the RCOphth mails-lists. PN performed the primary data analysis. $L M, P N$ and D'OB were involved in the interpretation of the results. LM and PN drafted the paper, which was reviewed and revised by all authors. LM and PN are accountable for the data integrity. All authors approved the final paper.

\section{COMPETING INTERESTS}

DO'B holds non-commercial research grants with Rayner Ltd., Alcon Inc., and Avedro Inc. He is a consultant to Zeiss and Sparca Inc.

\section{ADDITIONAL INFORMATION}

Supplementary information The online version contains supplementary material available at https://doi.org/10.1038/s41433-021-01717-5

Correspondence and requests for materials should be addressed to L.M.

Reprints and permission information is available at http://www.nature.com/ reprints

Publisher's note Springer Nature remains neutral with regard to jurisdictional claims in published maps and institutional affiliations. 